\title{
Analyse de l'activité d'élevage bovin et transformation des systèmes de production en situation sylvopastorale algérienne
}

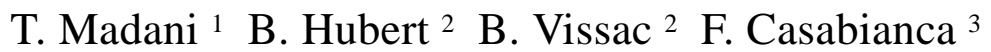

\begin{abstract}
Mots-clés
Bovin - Performance de reproduction - Etat corporel Performance animale - Méthode d'élevage - Système sylvopastoral Algérie.
\end{abstract}

\begin{abstract}
Résumé
Dans cette étude a été analysé le rôle joué par l'élevage bovin dans les systèmes d'élevage et les exploitations d'un massif forestier du Nord-Est algérien; le massif est représentatif des 500000 ha de la suberaie algérienne qui constitue la partie la plus arrosée et héberge la majeure partie du cheptel bovin allaitant du pays. Après avoir présenté le cadre de l'étude, les sources d'informations et les types d'élevage, les auteurs ont traité l'élaboration des performances de reproduction et de croissance des bovins. Cette démarche méthodologique de l'analyse des systèmes d'élevage a insisté sur les interactions entre les profils de reproduction des femelles, le type de matériel animal utilisé et la dynamique de l'état corporel qui s'est avéré un indicateur fiable pouvant constituer un outil de diagnostic dans la maîtrise de la reproduction. La confrontation des pratiques avec l'évolution des besoins des animaux et des ressources pastorales a permis de repérer des périodes dites saison-pratique, afin d'analyser les ajustements mis en œuvre et leur incidence sur les performances. Aussi, l'étude des pratiques et de l'évolution des systèmes d'élevage a permis de produire des catégories de systèmes de production plus pertinentes et plus opératoires pour rendre compte des trajectoires en cours. Le présent travail a contribué ainsi à la production de cadres de références adaptés à la situation, pouvant servir sans avoir à recourir aux seuls paradigmes de l'intensification.
\end{abstract}

\section{INTRODUCTION}

Aujourd'hui comme hier, l'élevage des bovins à viande est contingent du rôle qu'il joue dans les exploitations et les systèmes agraires. Le coût élevé de ce bétail et la durée de son intervalle de génération le rendent particulièrement sensible au changement. $\mathrm{Si}$ la littérature comprend de nombreuses références expérimentales sur les conditions «techniquement rationnelles » de sa maîtrise, peu d'éléments concernent des contextes géographiques marginaux et des situations de crise. Les connaissances issues d'expérimentations sont difficilement utilisables sans une analyse des sys-

\footnotetext{
1. Institut de biologie, université de Sétif, BP 808, 1900 Sétif, Algérie 2. Inra-Sad, 147 rue de l'Université, 75338 Paris Cedex 07, France Tél. : +33 (0)142759317; fax : +33 (0)1 42759507 E-mail : hubert@avignon.inra.fr

3. Inra-Lrde, quartier Grossetti, 20250 Corté, France
}

tèmes complexes dans lesquels l'élevage est inséré, de la représentation que les éleveurs s'en font et de leurs capacités réelles d'intervention.

Dans l'approche des activités d'élevage présentes sur un massif forestier du Nord-Est algérien, les auteurs ont été confrontés aux limites des références expérimentales disponibles. Au sein des cheptels des exploitations, l'articulation générale entre les bovins, les ovins et les caprins, décrite par ailleurs (15), a montré des liens fonctionnels entre les trois espèces en matière de gestion de la trésorerie et d'affectation des ressources pâturées et stockées. Les résultats présentés ici ont concerné plus spécifiquement les bovins, en cherchant à répondre à la question suivante : comment organiser un conseil technique différencié selon les types d'éleveur et adapté à chaque condition d'exercice de leurs activités d'élevage ? Pour y parvenir, les auteurs se sont efforcés d'abord de représenter le fonctionnement des systèmes d'élevage, puis d'identifier le rôle joué par les bovins dans l'évolution des systèmes de production. 


\section{MATERIEL ET METHODES}

\section{Contexte de l'étude}

L'étude s'est inscrite dans un projet d'aménagement intégré d'une suberaie du Nord-Est algérien (le massif des Béni Salah) qui couvre 50 p. 100 des 35000 ha du massif ; le reste est constitué de maquis plus ou moins dégradés et de futaies de chêne zeen sur les versants Nord. Ce massif est représentatif des 500000 ha de la suberaie algérienne qui constitue la partie la plus arrosée du pays (un peu plus de $600 \mathrm{~mm}$ de précipitations annuelles) et héberge une grande fraction du cheptel bovin allaitant de l'Algérie. Ce projet a pris sa place dans une longue histoire dont les événements récents ont marqué l'utilisation de ce territoire : la colonisation (qui lui a conféré son statut officiel d'espace forestier), la guerre d'indépendance (qui l'a vidé de ses habitants, les Beni Salah), l'industrialisation et le développement d'une agriculture marginale (qui ont favorisé son repeuplement par de nouvelles populations d'éleveurs venus du Sud, les Ouled Bechih) et les désordres récents de natures religieuse et politique (qui l'ont à nouveau vidé de toute population et activité économique). Les implications de ces événements sur les conditions d'utilisation du territoire, sur les migrations et sur les termes de 1'échange économique ont fortement perturbé les pratiques sylvopastorales conduisant, là comme ailleurs en zone méditerranéenne (18), les plus opportunistes à exploiter la rente écologique du territoire.

Le projet d'aménagement de la suberaie a visé ainsi simultanément à l'aménagement forestier proprement dit et à la protection de la forêt contre l'incendie, à la gestion de la population de cerfs de Barbarie en voie de disparition et à l'intégration des systèmes d'élevage au développement du massif (à la fois pour des raisons économiques et de protection contre les incendies) $(3,4)$. La première phase de ce projet a permis d'initier des études sur ces différents points dont la plupart étaient mal connus (cartographie et état des forêts, situation des populations de cerfs, fonctionnement et productions des systèmes d'élevage).

Les travaux sur les systèmes d'élevage portent sur les interactions entre les trois espèces de ruminants élevées, en termes d'utilisation du territoire et de dynamique des systèmes de production $(14,15)$. Ils s'intéressent plus globalement à la recherche d'une intégration durable, à bénéfices réciproques, des activités d'élevage et forestières. Pour cela, les études réalisées s'appuient sur les concepts de système d'élevage (13), de pratiques (12) et de modèle de comportement de l'agriculteur pour l'action (10). Elles visent à décrire et à interpréter les pratiques des éleveurs, reliées à l'élaboration des performances (12). Dans cet article sont principalement présentés les résultats portant sur l'élevage bovin.

\section{La situation et ses dynamiques}

Cette dynamique étant décrite par ailleurs (15), seuls les principaux traits sont rappelés ici. Jusqu'au début des années 80, les facteurs essentiels de l'évolution de ces exploitations correspondaient aux ressources du massif (importantes du fait de l'absence de leur utilisation pendant une dizaine d'années, correspondant à l'interdiction de résidence au sein du massif pendant la guerre d'indépendance) et aux capacités de développement de l'effectif du troupeau bovin ${ }^{1}$. Mais cette situation s'est transformée, du fait du développement propre des systèmes de production et des évolutions du contexte politique et socio-économique du pays. On peut distinguer deux voies selon la localisation :

- en forêt, l'enclavement, le statut précaire des terrains et l'absence d'équipement limitent le développement à la valorisation des ressources spontanées et à l'augmentation de la taille des troupeaux ; toutefois le niveau des ressources disponibles diminue ou, tout au moins, les ressources abondantes se situent plus loin des zones habitables (clairières et lisières du massif) ;

- en périphérie, la capitalisation, assurée avant 1980 grâce aux ressources pastorales du massif, s'est confrontée aux limites des structures d'exploitation (terre, travail et capital), de la taille des troupeaux et des types génétiques existants, favorisant l'orientation vers un modèle associant les cultures, l'élevage et l'engraissement.

Cette dernière situation n'est pas sans risques et ces structures restent fragiles et très dépendantes, d'une part, des fluctuations de prix des animaux maigres (puisqu'ils sont amenés à en acheter aux éleveurs de forêt) et, d'autre part, des ruptures d'approvisionnement ou des cours élevés des concentrés ${ }^{2}$. Ceux qui ont résisté à ces perturbations sont ceux qui ont conservé un troupeau de petits ruminants (15).

Quoi qu'il en soit de ces évolutions contrastées et de leurs ruptures, le bovin reste l'espèce pilote de ces systèmes d'élevage. C'est sur la production bovine que repose la mise en œuvre de stratégies d'équipement et de construction de bâtiment en dur (permettant l'engraissement ou la création d'un atelier laitier), voire pour des investissements immobiliers en ville. Sauf dans les petits élevages de subsistance, le bovin ne sert pas à assurer les besoins de trésorerie de la famille. Ainsi, en termes de commercialisation, on observe, selon les systèmes, des situations conjoncturelles de vente de mâles souvent âgés et maigres jusqu'à des stratégies d'engraissement visant des marchés particuliers. Les vêlages, regroupés en fin d'hiver, permettent non seulement aux veaux de profiter de la période de fortes disponibilités pastorales au printemps, mais également la reconstitution des réserves et le retour en chaleur du maximum de mères. La complémentation est réservée prioritairement aux vaches mères, quelquefois aux brebis mais jamais aux caprins.

\section{Sources d'information}

Trois sources d'information ont été utilisées dans l'étude relative à l'élevage des bovins :

- une enquête semi-directive (réalisée en 1989) sur la structure, le fonctionnement et les caractéristiques des systèmes d'élevage d'un échantillon de 67 exploitations jugées représentatives de différentes situations dans le massif ou à sa périphérie, la plupart concernées par les trois espèces de ruminants ; l'objectif a été de caractériser la diversité des systèmes de production et de l'organiser à partir de quelques types ;

- un suivi mensuel (1990-1992) de la reproduction, des variations d'effectif (naissances, morts, ventes et achats d'animaux), des choix d'orientation productive (vente précoce ou tardive, mise à l'engraissement, etc.) et des calendriers de pâturage des troupeaux d'un sous-échantillon de 12 exploitations stratifié parmi ces types ; le format - au sens de Vissac (22) - de chaque vache a également été noté (6) ;

- une mesure de croissance des jeunes et d'état corporel des mères - à l'aide de la grille d'appréciation par maniements corporels proposée par Agabriel et coll. (1) - à des périodes clés du cycle annuel (début de la complémentation en automne, fin de la période des mises bas et période de pic des saillies).

\footnotetext{
${ }^{1}$ Rares sont les éleveurs qui ont commencé l'élevage avec leur propre capital. La majorité d'entre eux a démarré avec une ou deux vaches en « confiage » appartenant à des parents résidant en ville. Petit à petit ces troupeaux se sont accrus profitant du capital pastoral disponible et d'un bon rapport de prix pour les produits carnés.

${ }^{2}$ Ils ont bénéficié à l'origine de prix des concentrés relativement bas, du fait d'une politique de subvention de ces aliments en raison de leur rôle stratégique pour le développement de l'élevage laitier et avicole.
} 


\section{RESULTATS}

\section{Typologie des exploitations}

La première enquête a permis de distinguer six types d'élevage à partir d'une analyse factorielle des correspondances prenant en compte les quatre premiers facteurs de l'analyse qui expliquent 38 p. 100 de l'inertie.

\section{Elevages pastoraux de plaine ou en lisière de la forêt}

Les trois espèces de ruminants (moins de 10 vaches et 20 à 40 mères de petits ruminants) étaient présentes dans ces élevages. Il n'y avait ni équipement, ni bâtiment en dur et la main d'œuvre y était faible. Les animaux étaient gardés sur les terres de l'exploitation (moins de 10 ha). Ils utilisaient des pâturages au printemps, les chaumes en été et vivaient des ressources de la forêt, de foin et d'aliments complémentaires (bovins) le reste du temps. La commercialisation était largement dépendante des besoins de trésorerie.

\section{Elevages pastoraux de forêt}

Ces élevages étaient caractérisés par l'absence d'équipement, de bâtiments en dur et par les trois espèces de ruminants (10 à 20 vaches et 40 à 60 brebis et chèvres) qui pâturaient l'espace collectif boisé et de petites surfaces de clairières. Celles-ci étaient louées à l'administration forestière et servaient d'appoint fourrager aux ovins et aux bovins en période de disette (automne-hiver). La commercialisation des produits était diversifiée : si la vente des chevreaux était principalement vouée à la trésorerie de la famille, d'autres ventes d'animaux étaient destinées à permettre l'achat de stocks fourragers.

\section{Elevages soutenus de plaine}

Situés en lisière de la forêt, ces élevages (10 à 20 vaches et plus de 60 brebis) pratiquaient également d'autres activités agricoles et non agricoles. Ils disposaient, outre une forte disponibilité en main d'œuvre (2 à 3 UTH ${ }^{1}$ permanents), de surfaces cultivées importantes (15 à 50 ha) - pour partie en propriété, pour partie louées ainsi qu'un bâtiment construit servant notamment à l'engraissement des taurillons. Ces unités se suffisaient à elles-mêmes pour les fourrages et partiellement les grains : les animaux pâturaient en forêt et étaient complémentés l'automne et l'hiver ; ils utilisaient les jachères au printemps et les chaumes de céréales en été. Une politique de renouvellement des femelles et de commercialisation des taurillons à deux ans, après trois à quatre mois d'engraissement, s'est clairement manifestée ici.

\section{Elevages soutenus de forêt}

Ces élevages, mobilisant aussi une forte main d'œuvre (2 à $3 \mathrm{UTH}$ ), exploitaient des terres de pâturage (5 à 15 ha) louées à l'administration forestière et achetaient du grain, mais ils n'avaient ni équipement, ni bâtiment en dur. Ces ressources étaient utilisées pour complémenter, à l'automne et en hiver des troupeaux ovins et bovins relativement importants (respectivement 40 à 60 et 20 à 30 femelles). Ils pâturaient le reste du temps les surfaces boisées. En plus du saisonnement des mises bas (hiver), une stratégie évolutionniste, équivalente à la précédente, a commencé à se mettre en place : politique de renouvellement, engraissement partiel des taurillons vendus selon des stratégies définies, la commercialisation des ovins répondant plutôt à l'équilibre de la trésorerie.

\section{Elevages de subsistance}

Situés en forêt et en périphérie avec une faible disponibilité en main d'œuvre, ces élevages étaient caractérisés par une absence d'équipement et de terres (utilisation des terres collectives et des

\footnotetext{
${ }^{1}$ Unités de travail humain
}

chaumes). Ils comportaient moins de cinq vaches ou quelques petits ruminants (moins de 30 mères) et dépendaient largement des aliments achetés. Leur stratégie de vente était très liée aux besoins de trésorerie.

\section{Gros élevages pastoraux}

Il s'agissait d'élevages de bovins (30 à 40 mères) et de caprins (40 à 70 chèvres) où, en dehors du pâturage permanent en forêt, les bovins étaient complémentés avec du foin de prairie naturelle (5-10 ha) produit sur l'exploitation. Dans cette conduite autonome et extensive, les jeunes animaux étaient vendus à la fin du printemps et en été, dès que leur état corporel le permettait.

A ces six types d'élevage, il fallait ajouter des unités de polyculture spécialisées dans l'engraissement de taurillons âgés (18 à 24 mois), situées en plaine, qui complétaient les ressources alimentaires de la culture de céréales et de fourrages, voire des résidus de maraîchage, par l'achat de concentrés. Pour les suivis de performances des troupeaux bovins, les auteurs se sont limités aux quatre premiers types décrits, qui dans la forêt et sa lisière, ont constitué les seules formes d'organisations ayant montré une certaine viabilité (15).

\section{Performances des troupeaux bovins}

\section{Fécondité des troupeaux et croissance des veaux}

Les informations sur les animaux ont été rapportées à la période de vêlage qui a été située, pour une année donnée (1990, 1991 et 1992), du premier octobre de l'année précédente au premier octobre de l'année correspondante. Les vaches n'ayant pas mis bas à cette date ont été considérées comme non gravides cette annéelà. Les produits commercialisés, issus de ces vêlages, ont été identifiés à la naissance ; l'âge minimum de vente des plus jeunes maigres a été de 8 mois.

Les naissances ont été saisonnées et sont intervenues de janvier à mai dans 90 p. 100 des cas, quels qu'aient été les systèmes d'élevage (figure 1), tant pour les primipares que pour les multipares, avec de légers décalages moyens entre années. Les vêlages hors saison (10 p. 100) ont eu lieu dans les systèmes d'élevage dont le soutien alimentaire a autorisé le retour des chaleurs en fin d'été et en automne, ce qui correspond aux résultats observés en Corse (5).

D'après les résultats du tableau I, le taux de mises bas moyen, tous élevages confondus, a été de 56 p. 100 (femelles âgées de deux ans et plus). Ce taux est passé à 65 p. 100 pour les femelles multipares (quatre ans et plus).

Ces résultats, conformes à ceux signalés au Mexique (7), ont été très supérieurs à ceux rapportés par Landais (11) et Lhoste (13)

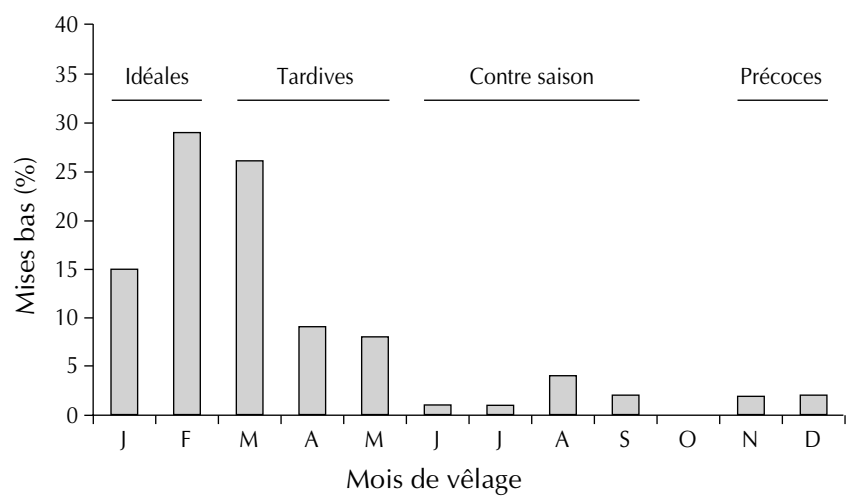

Figure 1 : étalement des mises bas. 


\section{Tableau I}

Taux de mises bas des femelles de plus de deux ans

\begin{tabular}{lcccc|} 
Type d'élevage & $\begin{array}{c}\mathbf{1 9 9 0} \\
(\mathbf{\%})\end{array}$ & $\begin{array}{c}\mathbf{1 9 9 1} \\
\mathbf{( \% )}\end{array}$ & $\begin{array}{c}\mathbf{1 9 9 2} \\
\mathbf{( \% )}\end{array}$ & $\begin{array}{c}\text { Moyenne } \\
\mathbf{( \% )}\end{array}$ \\
\hline $\begin{array}{l}\text { Soutenu de plaine } \\
\text { Soutenu de forêt }\end{array}$ & 84 & 45 & 60 & 62 \\
Pastoral de forêt & 57 & 53 & 56 & 55 \\
Pastoral de plaine & 47 & 68 & 41 & 56 \\
Moyenne & 62 & 52 & 53 & 50 \\
& & & & 56
\end{tabular}

dans les zones sahélo-soudaniennes africaines $(0,40)$ ou par Tourrand (20) dans la région du delta du fleuve Sénégal $(0,33)$, qui se rapprochent de ceux des élevages pastoraux de plaine. Les variations observées ici, entre années et systèmes d'élevage, se sont situées dans la fourchette des résultats de ces auteurs. C'est pour les élevages soutenus de forêt qu'elles ont été les moins fluctuantes. Malgré une moyenne équivalente sur la période observée, les élevages pastoraux de forêt ont montré d'importants écarts, probablement en liaison avec les fluctuations de la ressource fourragère. En revanche en plaine, le système soutenu et le système pastoral ont montré une différence considérable de taux moyens des mises bas. Les fluctuations observées sur les troupeaux des élevages soutenus de plaine ont été plus étonnantes et pourraient traduire les risques pris en faisant appel à des races améliorées ou en délaissant le troupeau allaitant au profit d'un deuxième troupeau réservé à la traite. La productivité numérique globale a été de 53 p. 100 en moyenne, le taux de mortalité signalé a été faible (2,7 p. 100).

Les veaux ont atteint un poids moyen de $140 \mathrm{~kg}$ à un an ; la croissance moyenne correspondante a été inférieure à $300 \mathrm{~g}$ par jour. Elle a évolué, pour les mâles, de $572 \mathrm{~g}$ jusqu'à six semaines à $250 \mathrm{~g}$ au-delà, mais l'écart-type a été élevé, une fois passée la période printanière : $150 \mathrm{~g}$ de six mois à un an. Ceci a suggéré une influence de la complémentation. Elle s'est fortement manifestée pour les mâles des élevages soutenus (de plaine ou de forêt), souvent croisés laitiers, dont la croissance a débuté à $720 \mathrm{~g} / \mathrm{j}$ pour tomber à $230 \mathrm{~g} / \mathrm{j}$ jusqu'à six mois et remonter à $370 \mathrm{~g} / \mathrm{j}$ en automne et en hiver. Les mâles des élevages pastoraux stricts (de race locale dominante) ont débuté au contraire à $350 \mathrm{~g} /$ jour, puis se sont maintenus jusqu'à six mois à $288 \mathrm{~g} / \mathrm{j}$, mais ils se sont effondrés par la suite en l'absence de complémentation ( $93 \mathrm{~g} / \mathrm{j}$ de six mois à un an). Ces écarts énormes ont donc cumulé des différences de conduite et de types génétiques.

\section{Analyse longitudinale des profils reproductifs des femelles}

Au vu de l'importance et de la variabilité des écarts du rythme annuel de reproduction des femelles, les auteurs se sont intéressés à la prise en considération de tranches continues de carrières reproductives individuelles. C'est ce qui a été fait de 1986 à 1992 en complétant les informations du suivi par celles rapportées de mémoire par les éleveurs avant 1990. Ont été éliminées de cette étude les vaches vendues pour des raisons affichées d'infertilité ou de troubles graves : cette proportion a été curieusement faible (2,2 p. 100). Ceci traduirait le fait que, dans ces situations, n'ont été interprétées comme échecs de reproduction que les femelles ayant subi plus de deux cycles annuels improductifs successifs. L'infertilité momentanée (inférieure à deux années successives) était donc vécue comme une régulation normale, alors que si elle durait plus longtemps elle devenait anormale. Cinq profils de mises bas ont été ainsi identifiés sur des périodes de cinq années continues (tableau II).

Près de la moitié des vaches n'ont mis bas qu'une année sur deux ou moins. La figure 2 traduit clairement une évolution croissante des dysfonctionnements depuis le système soutenu de plaine, où il n'a pas été n'observé de manques sur deux années consécutives, jusqu'aux deux systèmes forestiers, puis au système pastoral de plaine, où le standard tempéré de vêlage annuel quasiment régulier a été absent. Les quatre situations ont été représentées dans les deux systèmes d'élevage en forêt sans que les échantillons (25 à 30 vaches par système) n'aient permis de mieux les différencier. On peut relier globalement ces observations aux écarts concernant les disponibilités de ressources pâturées, de compléments distribués et de risques pris dans l'introduction d'animaux de format plus élevé selon les systèmes d'élevage.

A cet égard, Casabianca et Piccinelli (6) suggèrent que l'état du « système vache mère » au moment de la fécondation potentielle détermine un décalage plus ou moins accentué de la période de mise bas suivante. Ce décalage peut conduire, dans les cas extrêmes observés en Corse, à sauter un cycle annuel après

Tableau II

Profils de mise bas identifiés dans les élevages suivis

\begin{tabular}{|c|c|c|c|c|c|c|c|}
\hline \multirow[t]{2}{*}{$\mathbf{N}^{\circ}$} & \multirow[t]{2}{*}{ Désignation } & \multicolumn{5}{|c|}{ Occurrence des mises bas par année } & \multirow{2}{*}{$\begin{array}{l}\text { Fréquence } \\
\qquad(\%)\end{array}$} \\
\hline & & 1 & 2 & 3 & 4 & 5 & \\
\hline 1 & Vêlages 3 années successives au moins & $\begin{array}{l}+ \\
+\end{array}$ & $\begin{array}{l}+ \\
+\end{array}$ & $\begin{array}{l}+ \\
+\end{array}$ & + & $\begin{array}{l}+ \\
+\end{array}$ & 40 \\
\hline 2 & $\begin{array}{l}\text { Vêlages } 2 \text { années successives } \\
\text { Jamais } 2 \text { manques successifs }\end{array}$ & + & $\begin{array}{l}+ \\
+\end{array}$ & + & + & $\begin{array}{l}+ \\
+\end{array}$ & 16 \\
\hline 3 & Vêlages 1 année sur 2 & + & & + & & + & 30 \\
\hline 4 & $\begin{array}{l}\text { Vêlages } 2 \text { années successives } \\
\text { suivies de } 2 \text { années de repos }\end{array}$ & $\begin{array}{l}+ \\
+\end{array}$ & + & & + & $\begin{array}{l}+ \\
+\end{array}$ & 4 \\
\hline 5 & Vêlages 1 année sur 3 au plus & + & + & & + & + & 10 \\
\hline
\end{tabular}


Vélage une année sur trois au plus

Vélage deux années successives suivies de deux années de repos

Vélage une année sur deux

Vélage deux années successives jamais deux manque successifs

Vélage trois années successives au moins

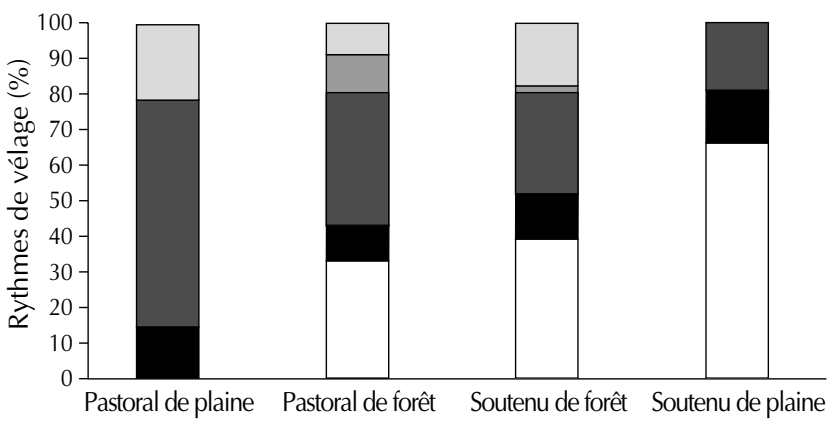

Figure 2 : rythmes de vêlages dans les quatre types d'élevage.

un vêlage tardif. Les auteurs ont essayé, en s'appuyant sur cette hypothèse, d'étudier la reproduction des vaches sur deux années successives. Cette façon de faire, qui ne prend pas en compte la mémoire de l'ensemble de la carrière des vaches mais mobilise les dépendances entre deux années successives, constitue une première approche de la question. Pour cette analyse, les deux types de systèmes pastoraux ont été regroupés. Le tableau III indique, pour chacun des trois types d'élevage, d'une part, les résultats connus de l'année n (effectifs, taux de vêlages et mois moyen de vêlage) et, d'autre part, les résultats correspondants des vêlages de l'année $n+1$ pour chacun des deux lots de vaches issues de l'année $\mathrm{n}$ selon qu'elles étaient parturientes ou au repos.

Il est apparu, dans les trois cas, que les taux de vêlages de l'année $\mathrm{n}$ ont globalement été maintenus l'année $\mathrm{n}+1$, le repos des femelles l'année $n$ ayant pratiquenıeni cunıensé, l'anmé $n+1$, le déficit de reproduction des vaches gravides l'année n. En ce qui concernait la répartition mensuelle des vêlages, le type soutenu de plaine a autorisé, d'abord, un étalement saisonnier sensiblement supérieur à celui des deux autres dont les vêlages ont été systématiquement concentrés sur le premier trimestre de l'année. Mais, dans tous les cas, les décalages de dates de vêlages ont été faibles à l'intérieur des types, même lorsque la saison a été plus étalée. Il est même apparu, surtout pour les femelles ayant mis bas deux années consécutives, une tendance au recyclage des vêlages en période favorable. Dans ce contexte, les régulations du système vache mère se sont donc plutôt traduites par des sauts de cycle annuel. Il est possible qu'il en soit autrement dans des situations moins drastiques où les phénomènes de stress ne correspondent pas aux mêmes amplitudes. En Corse, par exemple, cette situation ne se rencontre que dans les cas extrêmes, alors qu'ici ce phénomène a semblé se produire régulièrement.

\section{Age à la première mise bas}

En ce qui concernait le démarrage de la période productive, le premier vêlage est survenu entre deux et cinq ans, mais des différences importantes sont apparues entre systèmes d'élevage (figure 3), comme cela a été observé dans la région du delta du fleuve Sénégal (20). Dans les troupeaux soutenus de plaine, plus de la moitié des primipares ont mis bas dans la deuxième année et seulement 20 p. 100 sont restées improductives jusqu'à quatre ans, ce qui témoignait de l'attention accordée à l'élevage des génisses. En revanche, dans les systèmes pastoraux ou dans les exploitations de forêt pratiquant le soutien à la production, 70 à 80 p. 100 des vaches ont démarré leur carrière productive à partir de quatre ans. Ceci est cohérent avec le fait que, dans ces systèmes en équilibre financier précaire, les génisses étaient exclues de la complémentation en automne et en hiver, même quand celle-ci était assurée aux vaches. Les besoins de croissance des génisses ont été mal assurés, sauf en élevage soutenu de plaine, les formats adultes en ont été probablement influencés et le retard pris dans le démarrage de la reproduction a constitué une conséquence de ce déficit.

\section{Relations entre format, rythme d'élevage et état corporel}

Les types génétiques bovins et la stratégie de renouvellement des femelles sont marqués par les influences coloniales et intensificatrices passées. Ils résultent de la succession (sur la petite Brune de l'Atlas et sa branche locale la Guelmoise) de croisements avec des taureaux de races laitières et rustiques de format moyen (Tarine par exemple) jusqu'aux laitières de grand format, dont la Holstein constitue l'emblème le plus récent, aux Charolais et aux zébus. La diversité génétique des femelles résultant de ces métissages n'a pas été identifiable au plan de l'origine raciale. C'est pourquoi les auteurs ont préféré employer l'expression de format qui exprime la taille et la conformation de ces animaux issus du croisement de plusieurs types génétiques (22). Ces formats, correspondant à des poids ayant varié de 150 à $400 \mathrm{~kg}$, dans ces conditions, ont constitué ainsi l'indicateur le plus signifiant et pratique pour caractériser les vaches par grandes catégories (tableau IV).

Les variations d'état corporel n'ont pas été analysées pour les systèmes pastoraux de plaine ; la faiblesse de l'échantillon jointe aux conditions très extensives d'élevage situaient en effet une telle

\section{Tableau III}

Performances reproductives des vaches deux années consécutives

\begin{tabular}{|c|c|c|c|c|c|c|c|c|c|c|c|c|}
\hline \multirow[t]{3}{*}{ Type d'élevage } & \multirow[t]{3}{*}{ Effectif } & \multicolumn{3}{|c|}{ Année n } & \multicolumn{8}{|c|}{ Année $\mathbf{n}+1$} \\
\hline & & \multirow[t]{2}{*}{$\begin{array}{l}\text { Nom. } \\
\text { de } \\
\text { vêlages }\end{array}$} & \multirow[t]{2}{*}{$\begin{array}{c}\text { Taux } \\
\text { de } \\
\text { fécondité } \\
(\%)\end{array}$} & \multirow[t]{2}{*}{$\begin{array}{c}\text { Mois } \\
\text { moyens } \\
\text { de } \\
\text { mise bas }\end{array}$} & \multirow[t]{2}{*}{$\begin{array}{l}\text { Nom. } \\
\text { de } \\
\text { vêlages }\end{array}$} & \multirow[t]{2}{*}{$\begin{array}{c}\text { Taux } \\
\text { de } \\
\text { fécondité } \\
(\%)\end{array}$} & \multicolumn{3}{|c|}{$\begin{array}{l}\text { Dont vache } \\
\text { ayant vêlé } \\
\text { l'année n }\end{array}$} & \multicolumn{3}{|c|}{$\begin{array}{c}\text { Dont vache } \\
\text { n'ayant pas } \\
\text { vêlé l'année n }\end{array}$} \\
\hline & & & & & & & Nom. & Taux & Mois & Nom. & Taux & Mois \\
\hline Soutenu de plaine & 60 & 42 & 70 & 4,0 & 40 & 68 & 24 & 57 & 3,4 & 16 & 89 & 3,0 \\
\hline $\begin{array}{l}\text { Soutenu de forêt } \\
\text { Pastoral }\end{array}$ & 152 & 82 & 54 & 2,6 & 83 & 54 & 41 & 50 & 2,4 & 42 & 60 & 2,8 \\
\hline (plaine et forêt) & 91 & 50 & 55 & 3,2 & 50 & 55 & 16 & 32 & 2,0 & 34 & 83 & 3,3 \\
\hline
\end{tabular}


Ensemble des génisses suivies

Elevage soutenu de plaine

$n=22$

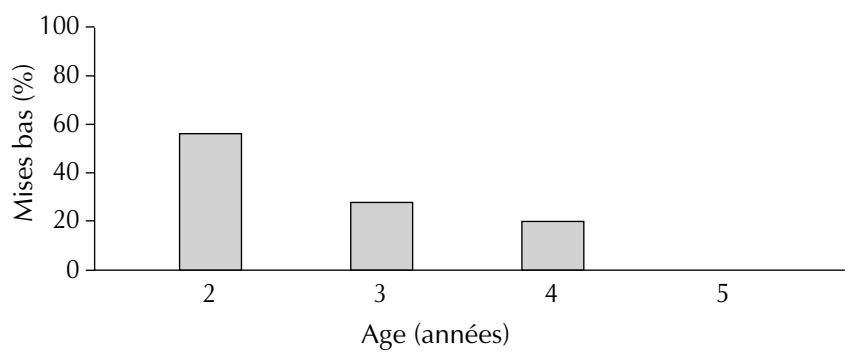

Elevage soutenu de forêt

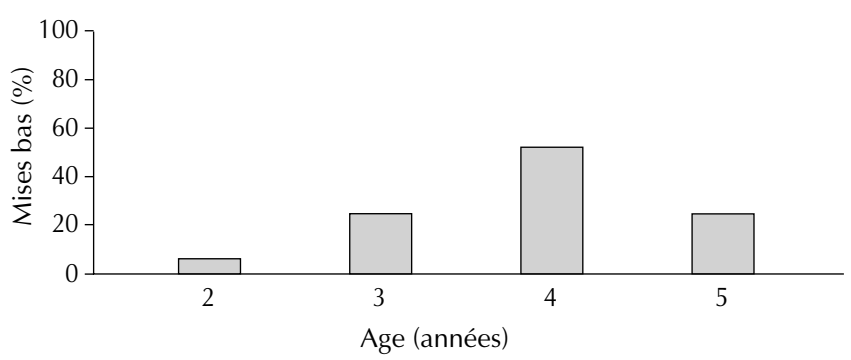

Elevage pastoral

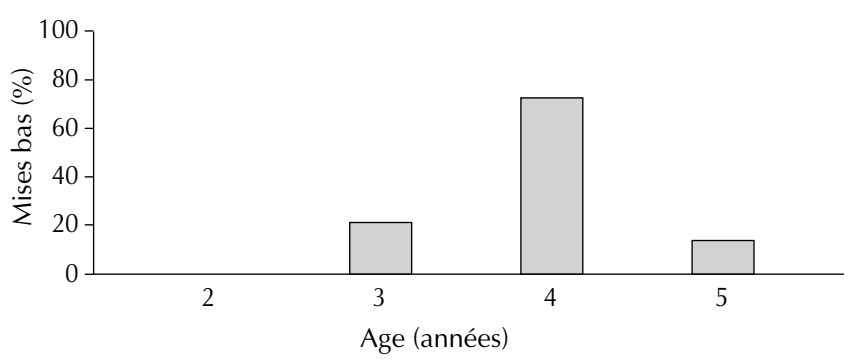

Figure 3 : répartition des âges à la première mise bas.

\section{Tableau IV}

Grille de classement du format des vaches et de leur équivalent pondéral

\begin{tabular}{ll} 
Format & Poids vif estimé $\mathbf{( k g )}$ \\
\hline Petit & $<200$ \\
Petit à moyen & 200 à 250 \\
Moyen & 250 à 300 \\
Moyen à grand & 300 à 350 \\
Grand & $>350$
\end{tabular}

analyse hors de propos. Les notes moyennes d'état corporel utilisées ont correspondu aux trois époques suivantes : mai 90 et mai 91 (pics de saillie) encadrant octobre 90 (début des complémentations éventuelles). Elles ont permis de mesurer l'état à la saillie, les variations d'état (mobilisation et restauration des réserves corporelles) et le retour à l'équilibre d'une année à l'autre au moment de la période sensible de reproduction des femelles (tableau V).

Si les variations moyennes ont été faibles, les différences ont été importantes entre les systèmes. Ces résultats ont fait apparaître, en premier lieu, la diminution de l'état corporel, entre le pic de saillies et le début de la complémentation, des femelles vides aussi bien qu'en lactation, dans les systèmes soutenus aussi bien que dans le système pastoral de forêt. En deuxième lieu, ils ont montré l'ampleur supérieure du processus de mobilisation-restauration des réserves corporelles chez les femelles en lactation des systèmes soutenus par rapport à celles qui ne l'étaient pas et par rapport à l'ensemble des femelles des systèmes pastoraux. En dernier lieu, ils ont indiqué une absence de récupération de l'équilibre initial à l'échéance du cycle annuel (au moins dans les conditions du mauvais automne 1990, voir plus loin).

Ces résultats ont été cohérents avec ceux relatifs aux performances de reproduction dans ces différents systèmes. Ils ont attesté de l'intérêt d'utiliser le paramètre dynamique de l'état corporel comme indicateur du pilotage de la reproduction des femelles, même sur de petits effectifs ( 15 à 25 femelles issues de plusieurs troupeaux de systèmes identiques). Néanmoins, les variations résiduelles d'états observées (écarts-type de 0,20 à 0,60 ) nécessitaient une analyse plus poussée des différences individuelles, en liaison notamment avec le format.

La variabilité est restée importante en forêt où, pourtant, les types génétiques locaux ont dominé, mais elle a différencié (figure 2) les systèmes pastoraux, où les reproductrices ont été moins soignées, et les systèmes soutenus. De plus, à l'intérieur de ces deux types, le rythme de reproduction a baissé fortement dès que le format a augmenté (figure 4). Pour cette comparaison, ont été éliminés les formats extrêmes des systèmes pastoraux dont l'effectif d'animaux inférieur à 12 p. 100 correspondait à moins de 10 animaux.

Le rôle du croisement et l'absence de complémentation se sont conjugués à des degrés divers pour expliquer ces différences. Ceci a été cohérent avec les résultats expérimentaux cités dans la littérature sur la conduite de troupeaux de femelles croisées de première génération, saillies par des taureaux de race non améliorée, ou de troupeaux des femelles n'ayant pas repris un certain état $(8,16,23,24)$, mais, dans les conditions du terrain, les observations ont résulté également de l'effet des pratiques des éleveurs. Pour en rendre compte, la figure 5 représente la répartition des formats des femelles de chaque troupeau avec les pratiques de croisement et les quantités de concentré distribuées pour chacun des troupeaux suivis dans les quatre systèmes d'élevage (soutenus et pastoraux, de plaine et de forêt). Les élevages pastoraux (exploitations 4, 7 et 8) n'ont comporté que des femelles de format réduit à moyen recevant, par tête, 100 à $300 \mathrm{~kg}$ de concentré sur l'année. La répartition des formats a été très étalée, avec une dominante vers les formats moyens, pour les élevages soutenus de forêt (exploitations 1, 6 et 5), sans relation directe avec les niveaux de distribution de concentré qui ont varié de $500 \mathrm{~kg}$, pour l'élevage ayant le plus d'animaux de format moyen à grand, à plus d'une tonne par vache et par an, pour celui qui a conservé un tiers de vaches à petit format et qui a réalisé de meilleures performances de reproduction. Les élevages soutenus de plaine (exploitations 3, 9 et 11) ont été, en revanche, dominés par des femelles de grand format, issues de croisement laitier (Tarin, Montbéliard ou Holstein) ou par des vaches de ces races achetées, voire élevées en génisses dans les élevages d'origine. Dans tous les cas, 


\section{Tableau V}

Moyennes et variations des notes d'état corporel par type de système d'élevage

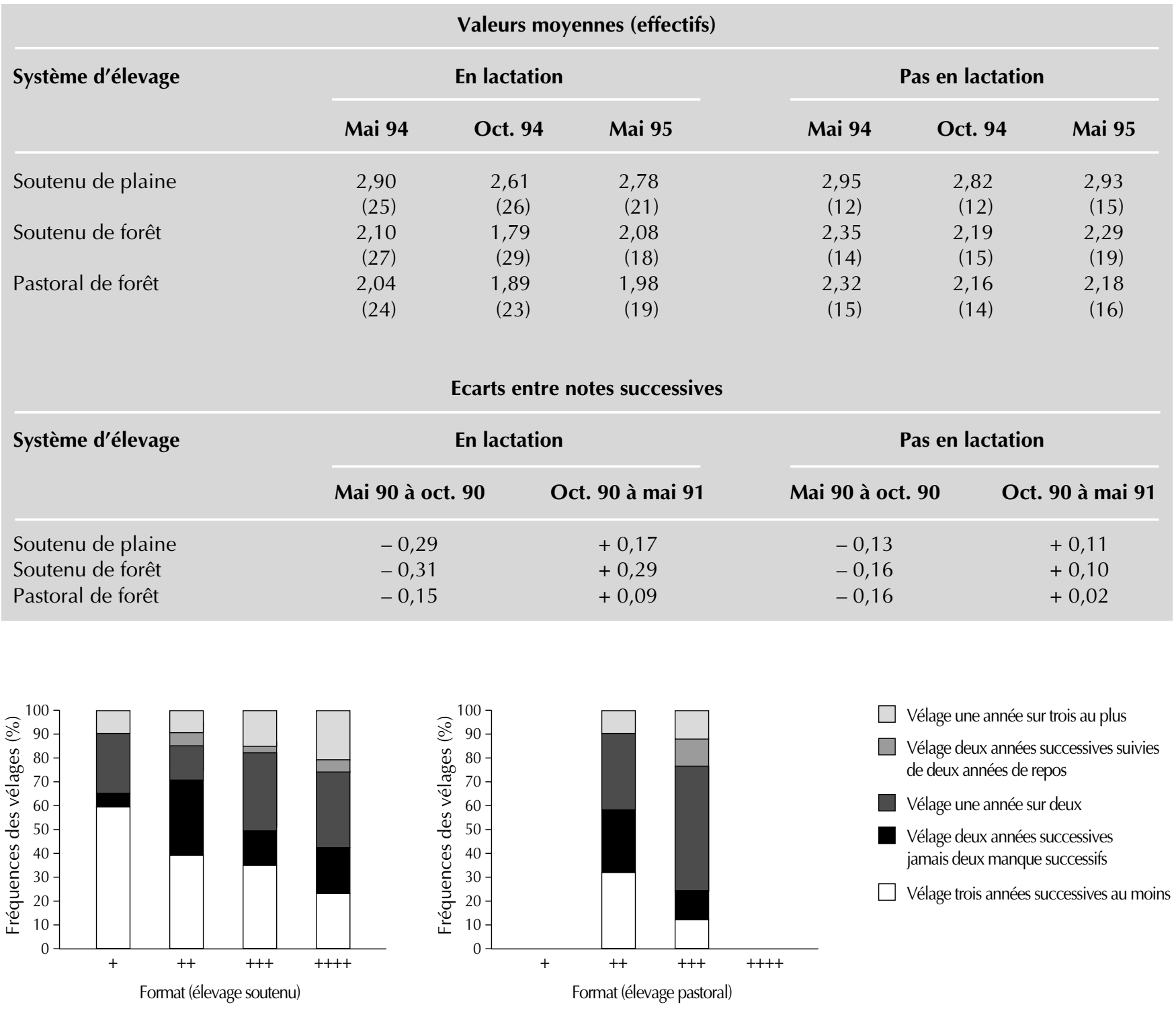

Figure 4 : relation entre format et rythme de vêlages dans les élevages de forêt.

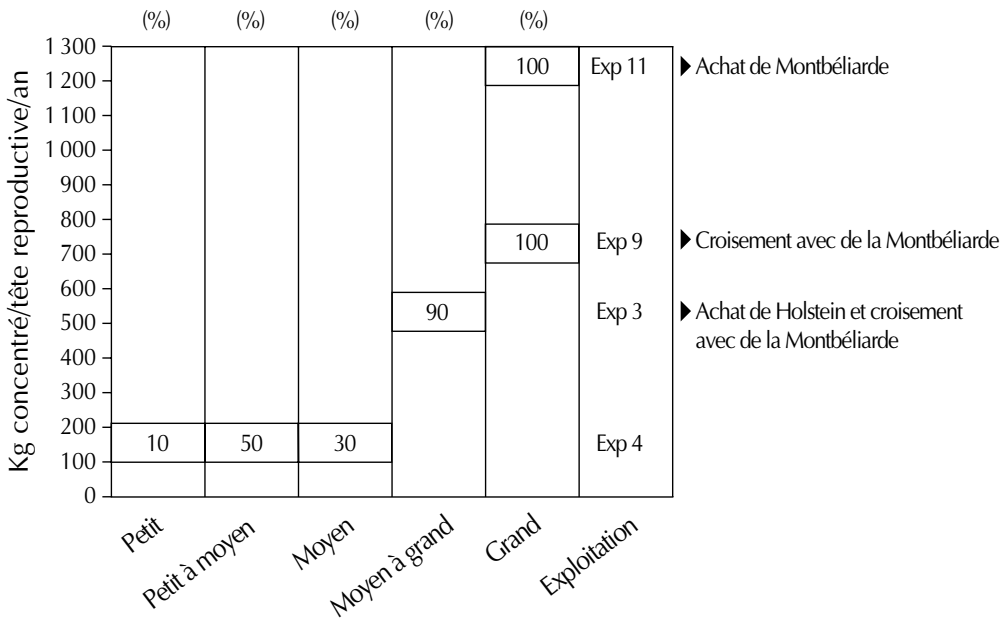

Exploitations (Exp) de plaine

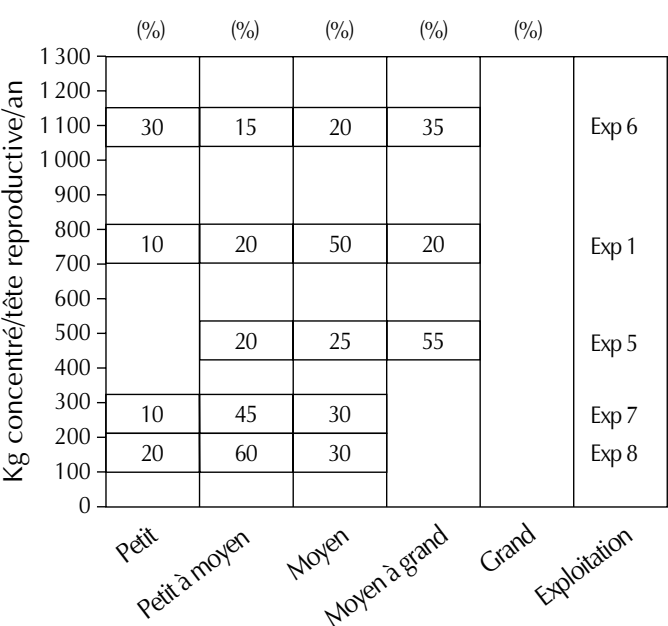

Exploitations (Exp) de forêt

Figure 5 : relation entre le format du troupeau (\%) et le niveau de complémentation. 
la complémentation a été supérieure, mais elle pouvait varier considérablement de 500 à $800 \mathrm{~kg}$, voire $1300 \mathrm{~kg}$, ce qui correspondait à une marge de variation conséquente en matière de stimulation nutritionnelle de la reproduction des femelles.

\section{Utilisation des ressources alimentaires}

Afin d'analyser les modes d'utilisation des ressources fourragères spontanées du massif selon une méthode qui permettait également de raisonner l'équilibration des ressources et des besoins des animaux, les auteurs se sont inspirés du concept de «saison- pratique », tel qu'il a été défini par Bellon et coll. (2), en référence à la notion de «fonction », préalablement caractérisée par Guérin et Bellon (9). La conduite des troupeaux peut être ainsi référée à quatre grandes saisons-pratiques, qui correspondent à des phases identifiées au cours du déroulement saisonnier, et à leurs relations tant au cours de la campagne annuelle qu'avec les campagnes précédente et suivante. On peut en outre distinguer deux périodes délicates à la soudure été-automne et au début du printemps.

Le tableau VI présente - pour ces six périodes et de manière synthétique - les pratiques et les objectifs d'ajustements alimentaires

\section{Tableaux VI}

Ajustements effectués dans la conduite alimentaire

\begin{tabular}{|c|c|c|c|c|}
\hline $\begin{array}{l}\text { Fonction } \\
\text { alimentaire }\end{array}$ & $\begin{array}{l}\text { Caractéristiques } \\
\text { des ressources }\end{array}$ & Elevages pastoraux & $\begin{array}{l}\text { Elevages soutenus } \\
\text { de forêt }\end{array}$ & $\begin{array}{l}\text { Elevages soutenus } \\
\text { de plaine }\end{array}$ \\
\hline $\begin{array}{l}\text { Soudure été- } \\
\text { automne : } \\
\text { Entretien des } \\
\text { mères en } \\
\text { lactation }\end{array}$ & $\begin{array}{l}\text { Pas de ressources } \\
\text { spontanées } \\
\text { assurées }\end{array}$ & $\begin{array}{l}\text { Distribution au mieux de } \\
50 \% \text { des besoins aux } \\
\text { vaches en mauvais état } \\
\text { pour leur permettre de } \\
\text { survivre en arrière saison }\end{array}$ & $\begin{array}{l}\text { Distribution au mieux de } \\
70 \% \text { des besoins aux } \\
\text { reproductrices afin de } \\
\text { maintenir leur état }\end{array}$ & $\begin{array}{l}\text { Distribution au mieux de } \\
100 \% \text { des besoins de } \\
\text { tout le troupeau }\end{array}$ \\
\hline $\begin{array}{l}\text { Automne : remise } \\
\text { en état des } \\
\text { vaches }\end{array}$ & $\begin{array}{l}\text { Repousses } \\
\text { aléatoires }\end{array}$ & $\begin{array}{l}\text { Seules les vaches } \\
\text { gravides ont été } \\
\text { concernées et ont reçu au } \\
\text { mieux } 30 \% \text { des besoins. } \\
\text { L'objectif a été d'éviter } \\
\text { une mobilisation } \\
\text { excessive des réserves } \\
\text { corporelles avant l'hiver }\end{array}$ & $\begin{array}{l}\text { Les vaches gravides, en } \\
\text { lactation et de gros } \\
\text { format ont été soutenues } \\
\text { (30 à } 100 \% \text { des besoins) } \\
\text { afin de maintenir leur état }\end{array}$ & $\begin{array}{l}\text { Selon les disponibilités en } \\
\text { herbe, les troupeaux ont } \\
\text { pu recevoir de } 0 \text { à } 100 \% \\
\text { des besoins }\end{array}$ \\
\hline $\begin{array}{l}\text { Hiver : fin de } \\
\text { gravidité }\end{array}$ & $\begin{array}{l}\text { Repousses de } \\
\text { l'automne } \\
\text { précédent, } \\
\text { ligneux, glands }\end{array}$ & $\begin{array}{l}\text { Seules les vaches en fin } \\
\text { de gravidité ont reçu } 20 \text { à } \\
40 \% \text { des besoins. Les } \\
\text { autres animaux ont utilisé } \\
\text { leurs réserves }\end{array}$ & $\begin{array}{l}\text { L'objectif a été de } \\
\text { maintenir l'état des } \\
\text { reproducteurs jusqu'au } \\
\text { printemps. Animaux } \\
\text { sensibles (gravides, en } \\
\text { lactation et en mauvais } \\
\text { état) : } 80 \text { à } 120 \% \text { des } \\
\text { besoins }\end{array}$ & $\begin{array}{l}\text { La totalité des besoins } \\
\text { ( } 90 \text { à } 120 \% \text { ) de tout le } \\
\text { troupeau est couverte par } \\
\text { le foin et le concentré }\end{array}$ \\
\hline $\begin{array}{l}\text { Début de } \\
\text { printemps : } \\
\text { allaitement des } \\
\text { veaux }\end{array}$ & $\begin{array}{l}\text { Ressources } \\
\text { aléatoires }\end{array}$ & $\begin{array}{l}1 \text { à } 2 \text { UF pour les vaches } \\
\text { en début de lactation }\end{array}$ & $\begin{array}{l}\text { Plus de } 70 \% \text { des besoins } \\
\text { des vaches en début de } \\
\text { lactation }\end{array}$ & $\begin{array}{l}\text { Utilisation possible de } \\
\text { parcelles d'orge ou de } \\
\text { prairies }\end{array}$ \\
\hline $\begin{array}{l}\text { Plein printemps: } \\
\text { allaitement des } \\
\text { veaux et remise } \\
\text { en état (saillie) }\end{array}$ & Pousse de l'herbe & $\begin{array}{l}\text { Au mieux, l'intervention } \\
\text { de l'éleveur corrige les } \\
\text { effets d'une mobilisation } \\
\text { excessive en hiver chez } \\
\text { les seuls reproducteurs }\end{array}$ & $\begin{array}{l}\text { L'objectif a été } \\
\text { modulable en fonction de } \\
\text { I'état des animaux à la } \\
\text { sortie de l'hiver et de la } \\
\text { qualité du printemps } \\
\text { (remise en état pour } \\
\text { supporter l'hiver prochain } \\
\text { ou saillir le maximum de } \\
\text { vaches) }\end{array}$ & $\begin{array}{l}\text { Surfaces fourragères } \\
\text { suffisantes pour une } \\
\text { maîtrise de la production } \\
\text { (saillies) }\end{array}$ \\
\hline $\begin{array}{l}\text { Eté : entretien des } \\
\text { mères allaitant } \\
\text { leurs veaux }+ \\
\text { croissance des } \\
\text { veaux }\end{array}$ & $\begin{array}{l}\text { Ligneux en forêt, } \\
\text { chaumes de } \\
\text { céréales }\end{array}$ & Aucune intervention & $\begin{array}{l}\text { Distribution des résidus } \\
\text { de fauche pour réaliser } \\
\text { les saillies d'été. Décision } \\
\text { de soutien alimentaire en } \\
\text { fonction du déficit en } \\
\text { production pastorale }\end{array}$ & $\begin{array}{l}\text { Soutien en concentré dès } \\
\text { le début de la } \\
\text { dégradation de la qualité } \\
\text { des chaumes }\end{array}$ \\
\hline
\end{tabular}


selon les grands types de systèmes d'élevage ; les systèmes pastoraux de plaine et de forêt y ont été regroupés. Les attentes du territoire du massif ont été bien différenciées d'un type de système à l'autre, ce qui n'était pas sans conséquence sur l'implication qu'ils pouvaient avoir dans l'aménagement du massif et l'entretien des espaces forestiers, dans la finalité de la prévention des incendies par un cloisonnement du massif et un meilleur contrôle de la végétation arbustive.

- Période de soudure entre l'été et l'arrière-saison : entretien des mères en lactation

La constitution des stocks de foin est une garantie essentielle des éleveurs vis-à-vis des incertitudes climatiques de l'automne et de celles de l'approvisionnement en concentré (prix et disponibilités). En fait, le niveau des pluies d'automne permet d'avoir une idée sur l'offre pastorale prévisible et de guider la stratégie de mobilisation des stocks compte tenu de l'état des animaux à cette date. En 1990, après une période de printemps-été déficitaire en ressources pastorales, les niveaux de soutien en foin et en grains pendant la soudure ont été très différents d'un système à l'autre. Ils ont été réduits en 1991, après un printemps-été plus favorable, permettant un meilleur état des animaux.

- Période automne-hiver : reconstitution des réserves et fin de gestation

Les deux campagnes 1990 et 1991 ont présenté un contraste exemplaire. La première a bénéficié de fortes pluies à l'automne 1989 permettant, à la différence de la seconde marquée par un déficit végétatif à l'automne 1990, un report hivernal de « stocks sur pied », alors que les ligneux, déjà utilisés depuis l'été, ont offert quelques ressources qui se sont dégradées progressivement en quantité et en qualité jusqu'au printemps suivant. En situation favorable, les élevages pastoraux ont fourni aux vaches, à l'automne, un complément de foin correspondant à 10-30 p. 100 des besoins alimentaires. Dans les élevages soutenus de forêt, l'apport complémentaire a été à la fois plus élevé (de 30 à 95 p. 100) et de nature variable (foin ou concentré) selon la qualité et la disponibilité des ressources pastorales, des stocks et de la trésorerie. Avec l'avancement de la saison automnale, le soutien alimentaire a été modulé par la qualité de la repousse et de la glandée. L'arrivée de l'hiver a correspondu à la phase de besoin maximal de fin de gestation et de début de lactation des premières mères allaitantes. En élevage pastoral, le soutien alimentaire a été réservé aux animaux les plus sensibles dans les deux premiers mois de lactation. Dans les autres systèmes d'élevage, le soutien complémentaire a varié autour du niveau des besoins globaux dès que les ressources fourragères se sont dégradées en quantité et en qualité (stratégie dite préventive par les éleveurs).

En situation défavorable, l'absence ou le retard des pluies interdisant le développement végétatif automnal, les éleveurs considéraient que l'hiver débutait dès le mois d'octobre. Mis à part l'émondage des branches de chêne zeen, les glands en forêt et les rameaux d'olivier en lisière, l'alimentation animale dépendait totalement de la complémentation. Les éleveurs productivistes de plaine, souvent confortés par leur appartenance à des coopératives, pouvaient compléter facilement leur stock de foin par des concentrés que les éleveurs de forêt n'obtenaient, en revanche, qu'à des prix souvent exorbitants sur le marché parallèle. Ces derniers réservaient alors la complémentation aux vaches et aux veaux de l'année, et les génisses et les taurillons recevaient une stricte ration de survie. Ils étaient souvent conduits, à cette occasion, à décharger leurs animaux maigres vers des ateliers d'engraissement dans des conditions de prix très défavorables.
- Ajustements de printemps : assurer l'allaitement des veaux et la saillie

Les deux campagnes ont été très contrastées : le printemps 1990 a été précoce, mais court (début mars à fin mai), alors qu'en 1991 il s'est traduit par un début de pousse d'herbe en mars, suivi d'une explosion végétative qui s'est prolongée d'avril en juillet. Ces différences et les pratiques correctives qui leur ont été associées ont eu des conséquences importantes sur la reconstitution des réserves et la limitation de la durée de l'anœstrus post-partum. Le pâturage des repousses herbacées a été complété dans certains élevages de plaine par celui des parcelles de jachère, voire d'orge en vert en cas de besoin, ainsi que par une complémentation en concentrés. Le territoire agricole a alors été utilisé en début de saison ainsi qu'après la moisson. Ces pratiques ont reposé sur un allottement du troupeau distinguant les animaux selon leur degré de sensibilité, afin de leur affecter les ressources les plus appropriées à leurs besoins estimés.

Dans les élevages soutenus de forêt, ces pratiques ont été limitées par les contraintes collectives qui ont pesé sur l'utilisation des surfaces fourragères : les animaux à plus forts besoins ont été maintenus dans des clairières collectives ou des prairies de fauche individuelles où ils ont été complémentés en concentré. Les autres animaux ont utilisé des ressources de moindre qualité qu'ils ont eu à rechercher sur des surfaces étendues. Ces stratégies ont donc mis en jeu des anticipations fondées sur l'état des animaux et sur le devenir de l'utilisation des ressources des prairies aux périodes ultérieures (pâturage ou fauche pour stockage). Les élevages pastoraux n'ont pas disposé d'une telle gamme de ressources potentielles ni des moyens financiers d'accès aux aliments distribués, leur capacité d'adaptation a été beaucoup plus limitée et ils ont été beaucoup plus dépendants des conditions météorologiques de l'année, ce qui s'est constaté dans leurs résultats techniques.

- Ajustements en été : maintenir la croissance des veaux

La prolongation de la pousse printanière en 1991 a permis, pour l'ensemble des élevages, de réduire la période estivale et de reporter sur l'arrière-saison le stock de ligneux fourragers de l'année. Les troupeaux ont utilisé de l'herbe sèche jusqu'à la fin de l'été, ce qu'ils n'avaient pas pu faire en 1990. La stratégie développée dans cette période a été très liée en forêt à la succession de la pousse et de l'utilisation des stocks d'herbe sur pied puis des ligneux fourragers, les animaux ayant utilisé diversement ces ressources dans la journée selon l'intensité de l'ensoleillement. Cela a donné une certaine souplesse aux élevages soutenus de forêt et a expliqué le relatif allongement de leur période reproductive. Tel n'a pas été le cas des élevages de plaine dont la disponibilité et la maîtrise des espaces fourragers ont eu comme conséquences des vêlages et des fécondations plus précoces, moins dépendantes des disponibilités pastorales.

\section{DISCUSSION}

\section{Des systèmes d'élevage différenciés et évolutifs}

La maîtrise technique de l'élevage bovin dans ces systèmes sylvopastoraux suppose de prendre en compte une série de régulations conjointes. Elles s'expriment au cours de l'année et des générations animales et humaines et elles opposent souvent, dans les décisions, l'intérêt immédiat et le long terme (22). L'arbitrage des éleveurs est d'autant plus difficile que leurs marges de manœuvre sont réduites et que les pressions de l'environnement socio-économique peuvent être incitatives mais éphémères et risquées. On peut intégrer ainsi les régulations mises en œuvre de différentes manières. 
Il y a celles qui correspondent aux régulations biologiques entre les fonctions de la femelle (lactation, croissance, gravidité et reproduction). Elles se manifestent au cours du cycle annuel et déterminent, en région méditerranéenne (5), la capacité des femelles à restaurer leurs réserves après la mise bas d'hiver grâce à la pousse d'herbe printanière (17). Mais le type génétique est aussi important pour déterminer la façon dont la femelle arbitre entre ses différentes fonctions en situation de sous-nutrition. Elle met en jeu des équilibres qui se manifestent, eux, au cours de la carrière de la femelle. Vallerand (21) l'a montré au Cameroun sur les ovins Djallonké de petite taille : de petites différences génétiques $\left(1 / 8^{\mathrm{e}}\right.$ de sang de race européenne de format moyen) se traduisent sur les femelles croisées par des réductions fortes de longévité ${ }^{1}$.

Il y a celles mises en œuvre pour faire jouer aux espèces animales un rôle conforme à leurs caractéristiques biologiques pour valoriser les ressources fourragères et la trésorerie ; si le bovin reste l'espèce privilégiée pour un objectif à long terme, les ovins et les caprins jouent un rôle essentiel dans les situations les plus précaires pour assurer le maintien et le décollage du système d'élevage dans son ensemble et de sa fraction bovine en particulier. Dans certains systèmes, les ovins sont orientés vers un second atelier productif complémentaire d'un troupeau bovin de format moyen (15).

Enfin, il y a celles qu'imposent les contraintes du système d'élevage en matière de main d'œuvre, d'accès aux ressources des territoires ainsi que de trésorerie. Il est alors possible de reconsidérer la première typologie alliant des éléments fonctionnels et structurels pour établir de nouvelles catégories, plus pertinentes en termes de systèmes de production et plus opératoires pour rendre compte des dynamiques passées et des trajectoires en cours, permettant ainsi de distinguer les systèmes suivants :

- les élevages pastoraux, de plaine ou de forêt, qui n'ont pas accusé de transformations importantes et sont encore à un stade de précapitalisation, dont le cheptel est la ressource individuelle principale ;

- les élevages soutenus de forêt qui sont en montée productive disposant d'accès à des ressources alimentaires assurées leur permettant de soutenir la production ;

- les élevages soutenus de plaine qui sont dans une dynamique productiviste imitant des logiques d'intensification agricole qui les orientent vers un contrôle de la productivité individuelle des animaux.

Les principales caractéristiques de ces trois types de systèmes sont présentées dans le tableau VII.

Cette étude montre la possibilité de représenter le fonctionnement des systèmes d'élevage et d'en déduire des éléments de maîtrise adaptés à la diversité des situations. Le suivi de la dynamique de l'état corporel constitue, en particulier, un indicateur de pilotage d'autant plus utile qu'il rejoint la culture des éleveurs dans l'appréciation de leurs animaux et qu'il est mobilisé ici en tenant compte de leurs logiques d'élevage. Les auteurs n'ont malheureusement pas pu l'utiliser en situant les séquences de carrière des femelles étudiées sur l'ensemble de leur cycle vital et en identifiant la phase juvénile (génisses et premier vêlage) dans la séquence. On sait, en effet, que c'est au cours de cette phase que la compétition entre les fonctions physiologiques est la plus vive dans l'arbitrage de l'utilisation métabolique des aliments consommés, que réalisent les femelles selon leur aptitude génétique (21). Les retards de puberté et la diversité des modes relatifs de conduite des génisses et des vaches selon les systèmes d'élevage incitent à poursuivre un tel travail dans ce sens.

Ces données illustrent les différences de conception d'un système à l'autre et, en particulier, la difficile tension à laquelle sont soumis les élevages ayant augmenté le format des vaches mères, plus sensibles aux aléas des ressources spontanées et donc plus dépendantes de l'apport d'aliments concentrés pour réguler leurs variations d'état. Toutefois, comme il n'y a pas de connaissances bien établies pour ajuster cette complémentation, les pratiques sont très variables et probablement plus liées aux disponibilités monétaires des éleveurs qu'à un raisonnement technique établi. Ainsi, quand les éleveurs peuvent acheter du complément, les niveaux de distribution sont importants, voire très importants. Les moyens financiers proviennent fréquemment de la vente des produits des troupeaux de petits ruminants, principalement les ovins. Certains éleveurs ont ainsi réduit le troupeau bovin à quelques vaches de grand format et augmenté également le format des ovins pour en améliorer la productivité, ce qui leur permet de soutenir, par la vente de plus gros agneaux, une production régulière des vaches de grand format, en engraissant si possible eux-mêmes les veaux de façon à conserver la valeur ajoutée du produit final (15). On arrive ainsi à constater des situations assez paradoxales où des élevages, constitués petit à petit à partir d'une rente pastorale valorisée par des bovins de race locale, se sont ainsi progressivement transformés en élevage bovin allaitant, quasiment hors sol, avec des animaux de grand format, dont la réussite repose sur le maintien d'un élevage de petits ruminants qui sont, eux, encore susceptibles de valoriser les ressources spontanées du massif et de ses alentours !

\section{Méthodologie adaptée en situation}

Pour comprendre le sens de cette étude sur la conduite individuelle des troupeaux en situation sylvopastorale, il faut d'abord insister sur le poids des dynamiques lourdes que l'histoire a fait peser sur ces systèmes d'élevage : en premier lieu la dévalorisation de la rente pastorale (constituée au cours de la guerre d'indépendance), remise en cause dans le contexte d'une économie fondée prioritairement sur l'exploitation des ressources minérales. Les limites de ces perspectives sont apparues récemment avec la reconnaissance officielle d'un secteur agricole privé à côté des domaines autogérés qui avaient marqué le développement agricole algérien pendant plus de deux décennies. Ceci confirme la pertinence du choix des éleveurs de ce massif, même si les derniers événements politiques mettent de nouveau en cause la permanence des installations dans la zone. On peut imaginer que ces événements seront suivis d'une relance de l'élevage, dans un contexte économique plus ouvert.

Dans la période où l'étude a été menée, le poids de la dimension collective de l'environnement socio-économique s'est ainsi manifesté au sein de la filière en faveur de techniques d'élevage et de races exogènes qui se sont révélées peu adaptées à de tels contextes. Les effets de ces tendances évolutives ont été accentués en raison de la durée de l'intervalle de génération entre bovins et du rôle de «modèle » qu'ont joué les premiers innovateurs. Les éleveurs n'ont pas pu bénéficier d'une organisation de l'encadrement technico-économique et de la commercialisation qui auraient pu limiter ces inconvénients, pas plus qu'ils n'ont disposé de protection contre les dérégulations en cours des intrants et des produits de leurs systèmes de production. Ils ont dû alors se préoccuper de maîtriser eux-mêmes les aléas de systèmes d'élevage moins extravertis, sans négliger pour autant les dimensions collectives d'une telle maîtrise, qu'il s'agisse de l'utilisation des ressources du territoire, d'un accès économique aux circuits d'approvisionnement comme de commercialisation, et de la pression génétique en faveur d'animaux de plus gros format mais plus exigeants ${ }^{2}$. La

\footnotetext{
${ }^{1}$ Il traduit cela en image en comparant la courbe de croissance des femelles à la trajectoire de l'obus : les femelles croisées de format amélioré ont un tir vertical mais explosent en plein vol alors que les petites femelles locales au tir tendu horizontal prolongent bien leur carrière.

${ }^{2}$ C'est ce que le projet d'aménagement intégré aurait pu permettre s'il n'avait pas été remis en cause par les derniers événements politiques en Algérie.
} 
production de connaissances sur une telle situation vise à contribuer à l'élaboration de cadres de références, sans le recours aux seuls paradigmes de l'intensification.

Faute de connaissances établies utilisables pour le conseil dans une telle perspective, les auteurs ont produit une méthode d'analyse des pratiques des éleveurs pour qu'elle puisse être confrontée à leurs résultats techniques. Ils ont fourni des indicateurs qui peuvent être utilisés dans le pilotage des troupeaux et qui sont à la fois référençables expérimentalement et fondés sur des expertises inscrites dans la culture technique des éleveurs (estimations du format et de l'état corporel des vaches). Cela postule à donner un statut aux savoir-faire locaux et à les utiliser dans l'identification des actions de développement ; aussi, la méthodologie produite permet d'améliorer l'efficacité des interventions en ciblant les catégories d'animaux qui les requièrent et les périodes concernées.

L'adaptation des éleveurs aux crises des systèmes d'élevage bovin pose des questions qui se différencient selon les systèmes et la diver- sité des logiques correspondantes dans le cadre d'une organisation globale de la production et des techniques. Ainsi, d'une première typologie alliant des éléments fonctionnels et surtout structurels issus d'observation, les auteurs ont abouti, par une analyse d'indicateurs zootechniques variés, à de nouvelles catégories plus pertinentes en termes de systèmes de production. Elles sont de ce fait plus opératoires pour rendre compte des dynamiques passées et en cours, et plus adaptées, en définitive, au renouvellement du conseil et de la recherche zootechnique. Ces catégories distinguent des élevages de précapitalisation ne disposant pas assez de cheptel et ne réalisant pas des performances suffisantes pour amorcer le développement de leur élevage, des élevages en montée productive disposant assez d'animaux et de ressources individuellement appropriées et des élevages hors massif forestier qui peuvent développer des logiques d'intensification fondées sur la productivité individuelle.

Ces travaux complètent ceux déjà publiés par Madani et coll. (15) sur la conjonction entre espèces et les régulations particulières qui

\section{Tableau VII}

Caractéristiques de fonctionnement des systèmes d'élevage bovin

\begin{tabular}{|c|c|c|c|}
\hline Caractéristiques & $\begin{array}{l}\text { Elevage en } \\
\text { précapitalisation }\end{array}$ & $\begin{array}{l}\text { Elevage en montée } \\
\text { productive }\end{array}$ & Elevage productiviste \\
\hline $\begin{array}{l}\text { Evolution de l'effectif des } \\
\text { adultes }\end{array}$ & $\begin{array}{l}\text { Variable selon les projets des } \\
\text { éleveurs et les accidents }\end{array}$ & $\begin{array}{l}\text { En diminution pour cause } \\
d^{\prime} \text { investissements }\end{array}$ & Stable ou en progression \\
\hline \multicolumn{4}{|l|}{$\begin{array}{l}\text { Stratégies de } \\
\text { commercialisation }\end{array}$} \\
\hline $\begin{array}{l}\text { commercialısation } \\
\text { adultes (femelles de } \\
\text { reforme) }\end{array}$ & $\begin{array}{l}\text { En majorité sont morts à } \\
\text { I'exploitation }\end{array}$ & $\begin{array}{l}\text { Ont assuré une partie des } \\
\text { investissements }\end{array}$ & $\begin{array}{l}\text { Ont assuré une partie des } \\
\text { investissements }\end{array}$ \\
\hline jeunes & $\begin{array}{l}\text { Vendus à un âge variable } \\
\text { selon les besoins en } \\
\text { trésorerie, maigres à des } \\
\text { poids estimés à } 100-120 \mathrm{~kg} \\
\text { vif à } 18 \text { mois }\end{array}$ & $\begin{array}{l}\text { Vendus entre } 10 \text { et } 18 \text { mois, } \\
\text { maigres et/ou partiellement } \\
\text { engraissés, à des poids } \\
\text { estimés à } 150-200 \text { kg vif }\end{array}$ & $\begin{array}{l}\text { Vendus après finition à l'âge } \\
\text { de } 24-30 \text { mois, à des poids } \\
\text { estimés à } 300-600 \text { kg vif }\end{array}$ \\
\hline Age à la première mise bas & 4 ans & 4 ans & 2 à 3 ans \\
\hline Fécondité & 0,4 à 0,6 & 0,65 & 0,7 \\
\hline Productivité numérique & 0,4 à 0,6 & 0,5 à 0,6 & 0,5 à 0,7 \\
\hline Format moyen des vaches (kg) & 200 à 250 & 250 à 350 & 250 à 350 \\
\hline \multicolumn{4}{|l|}{$\begin{array}{l}\text { Proportions des rythmes } \\
\text { de reproduction (\%) }\end{array}$} \\
\hline profils $1+2$ & 0 à 30 & 35 & 80 \\
\hline profil 3 & 40 à 50 & 30 & 20 \\
\hline profils $4+5$ & 20 à 25 & 20 à 25 & 0 \\
\hline $\begin{array}{l}\text { Quantité de concentré acheté } \\
\text { par an et par reproductrice (kg) }\end{array}$ & 150 à 300 & 500 à 1000 & 450 à 1200 \\
\hline Evolution prévisible & $\begin{array}{l}\text { Selon l'impact des variations } \\
\text { de la production pastorale. } \\
\text { Les troupeaux ont été en } \\
\text { reproduction simple ou en } \\
\text { progression selon le rythme } \\
\text { de commercialisation }\end{array}$ & $\begin{array}{l}\text { Selon celle du rapport prix } \\
\text { du concentré/prix de la } \\
\text { viande et probablement vers } \\
\text { I'arrêt de l'intensification ou } \\
\text { I'amorce de l'extensification } \\
\text { du mode de conduite }\end{array}$ & $\begin{array}{l}\text { Selon la place et le rôle de } \\
\text { I'atelier bovin dans le projet } \\
\text { global de développement de } \\
\text { l'exploitation (ateliers laitier, } \\
\text { maraîchage, etc.) }\end{array}$ \\
\hline
\end{tabular}


en résultent, tant en matière d'affectation des portions de territoire que de calendrier de production, de gestion de la trésorerie des exploitations et de l'organisation de l'élevage. L'apport de cette étude sur l'élevage bovin conduit à plusieurs remarques. Dans ces systèmes plurispécifiques, le bovin est la seule espèce qui permet le maintien de l'exploitation ou l'amorce de son développement : les fonctions et logiques de l'élevage des petits ruminants s'infléchissent ainsi différemment mais toujours à son profit. Toutefois, les méthodes mises en œuvre sur les bovins peuvent servir pour étudier et maîtriser le pilotage des autres types de troupeaux conduits dans la même exploitation avec des logiques cohérentes. Les caractéristiques des ovins et des caprins, le rythme de reproduction plus rapide notamment, peuvent favoriser en retour des comparaisons expérimentales plus rapides face à la faible quantité de connaissances disponibles sur les voies de maîtrise des risques en élevage extensif.

\section{- CONCLUSION}

Plus généralement, ces recherches alimentent de façon concrète le débat sur les relations entre l'aménagement et la gestion des forêts et la conduite d'élevage, en précisant les interférences du régime pastoral avec la croissance et la pérennité de la végétation. La durabilité des systèmes d'élevage dépend bien plus de la maîtrise de quelques périodes critiques (soudures, reconstitution des réserves corporelles et réussite des saillies, choix des types de produits à commercialiser) que de l'exploitation d'un capital renouvelable. Elle ne peut pas, bien sûr, se raisonner à l'échelle individuelle et indépendamment des points de vue des autres acteurs du territoire, ici les forestiers, et des filières. Les règles d'accès aux ressources sylvopastorales, l'approvisionnement en aliments concentrés, la maîtrise de l'amélioration génétique des animaux constituent ainsi un ensemble d'actions collectives exigeant des formes de coordination entre différentes catégories d'acteurs. Elles nécessitent également un accompagnement institutionnel pour favoriser un développement de ces types d'élevage qui serait en accord avec la production forestière et sa protection contre les incendies.

Cette étude sur un élevage empiétant sur l'espace forestier met en évidence les dépendances sociales et spatiales des modes d'élevage en situation limitante. La palette des choix techniques s'y réduit avec le niveau de précarité des situations des éleveurs. La volonté permanente d'imitation des plus favorisés a toujours eu tendance, depuis quelques décennies, comme on a pu l'observer également dans les pays industrialisés, à minimiser les risques ainsi générés : gérer au mieux les ressources fourragères en s'adaptant aux besoins des animaux compte tenu du matériel génétique et non l'inverse - en ayant recours à des achats extérieurs n'est pas possible ici dans la majorité des cas. Une telle logique de conduite de l'élevage montre ses limites dès que ses effets sont évalués à des échelles pluriannuelles, ainsi que l'a montré Tichit (19) sur les troupeaux mixtes de lamas et d'ovins sur l'altiplano bolivien.

Ces travaux illustrent les conséquences du déphasage avec l'exploitation des ressources locales, accentuées par le renchérissement des compléments en concentrés. Dans ce contexte, l'inadaptation du matériel génétique s'exprime sur le pas de temps, à la fois des processus biologiques (renouvellement des troupeaux et évolutions des parcours) et des changements sociaux et économiques (choix politiques et recomposition des filières agroalimentaires). Le transfert aux pays en développement du modèle qui a marqué la modernisation technique de l'élevage bovin des pays développés (animaux spécialisés de grand format) fait apparaître clairement les limites de la dépendance de ces animaux aux ressources disponibles. Le besoin primordial de sélectionner des ani- maux de format adapté au milieu d'élevage auquel ils sont destinés repose sur une sélection à partir des ressources génétiques locales. On est ainsi conduit à mieux respecter l'articulation entre les deux termes du couplage (ressources fourragères et génétiques) et les échelles temporelles de leur impact sur la régulation des systèmes d'élevage.

\section{BIBLIOGRAPHIE}

1. AGABRIEL J., GIRAUD J., PETIT M., 1986. Détermination et utilisation de la note d'état d'engraissement en élevage allaitant. Bull. tech. Crzv Theix, Inra, $66:$ 43-50.

2. BELLON S., GIRARD N., GUERIN G., 1999. Caractériser les saisons pratiques pour comprendre l'organisation d'une campagne de pâturage. Fourrages, 158 : 115-132.

3. BNEF, 1992. Projet d'aménagement intégré du massif des Beni Salah, Wilaya de Guelma. Partie 2 : Production sylvicole; partie 4 : Protection du cerf de Barbarie. Alger, Algérie, Bureau national des études forestières, 125 p. et 65 p.

4. BNEF, 1992. Projet d'aménagement intégré du massif des Beni Salah, Wilaya de Guelma. Partie 3 : Aménagements sylvo-pastoraux. Alger, Algérie, Bureau national des études forestières, $174 \mathrm{p}$.

5. CASABIANCA F., 1988. Elevage bovin corse. Système d'alimentation et matériel animal. Greghe e rughjoni, 13.

6. CASABIANCA F., PICCINELLI G., 1996. Recherches sur la gestion des troupeaux de bovins allaitants en milieu méditerranéen à fortes contraintes : modélisation de la reproduction. In: Cattle in the Mediteranean Area. Rome, Italie, EAAP, p. 105-120. ( $\left.N^{\circ} 86\right)$

7. CERVANTES N., CHOISIS J.P., LHOSTE P., 1990. Etude de I'élevage bovin par suivi zootechnique : le cas de I'Etat de Colima (Mexique). In : Gibon A., Vallerand F. eds, Approche globale des systèmes d'élevage et étude de leurs niveaux d'organisation. Luxembourg, CEE Pub., p. 159-164.

8. GAUTHIER D., PETIT M., TERQUI M., MAULEON P., 1984. Sousnutrition et fertilité. In : Actes symp. franco-israëlien Le potentiel de reproduction des bovins et des ovins, 21-23 février 1984. Versailles, France, Inrad éditions, p. 105-124. (Colloque Inra $n^{\circ} 27$ )

9. GUERIN G., BELLON S., 1989. Analysis of the functions of pastoral areas in forage systems in the Mediterranean region. Etud. Rech. Sys. agraires Dév., 16 :147-156.

10. HUBERT B., 1993. Pastoralisme et territoire, modélisation des pratiques d'utilisation. Cah. Agric., $3: 9$-12.

11. LANDAIS E., 1983. Analyse des systèmes d'élevage bovin sédentaire du Nord de la Côte d'Ivoire. Maisons-Alfort, France, lemvt, 759 p. (Etudes et synthèses $n^{\circ} 9$ )

12. LANDAIS E., DEFFONTAINES J.P., 1988. Les pratiques des agriculteurs. Point de vue sur un courant nouveau de la recherche agronomique. Etud. rurales, $109: 125-158$.

13. LHOSTE P., 1986. L'association agriculture-élevage. Evolution du système agropastoral au Sine Saloum (Sénégal). Thèse Doct., Ina-PG, Paris, France, 314 p.

14. MADANI T., 1993. Complémentarité entre élevages et forêts dans I'Est algérien : fonctionnements et dynamiques des systèmes d'élevage dans le massif des Béni Salah. Thèse Doct., université de Montpellier II, France, 266 p.

15. MADANI T., HUBERT B., LASSEUR J., GUERIN G., 2001. Association des bovins, des ovins et des caprins dans les élevages de la suberaie algérienne. Cah. Agric., 10 : 9-18.

16. MIALON M., RENAUD G., KRAUSS D., MENISSIER F., 1996. Caractéristiques et variabilités de la reprise d'activité sexuelle postpartum des vaches allaitantes Charolaises en troupeau expérimental. Renc. Rech. Rumin., 3 : 175-178. 
17. PETIT M., 1978. Vaches nourrices. In : Alimentation des ruminants. Versailles, France, Inra Editions.

18. SANTUCCI P.M., 1991. Le troupeau et ses propriétés régulatrices, bases de l'élevage caprin extensif. Thèse Doct., université de Montpellier II, France, 85 p.

19. TICHIT M., 1998. Cheptels multi-espèces et stratégie d'élevage en milieu aride : analyse de viabilité des systèmes pastoraux camélidésovins sur les hauts plateaux boliviens. Thèse Doct., Ina-PG, Paris, France, $285 \mathrm{p}$.

20. TOURRAND J.F., 1993. Le développement de l'agriculture irriguée et la transformation de l'élevage dans la région du delta du fleuve Sénégal. Thèse Doct., université de Paris VI, France, 340 p.
21. VALLERAND F., 1979. Réflexions sur I'utilisation des races locales en élevage africain. Exemples du mouton Djallonké dans les conditions physiques et sociologiques du Cameroun. Thèse Doct., université polytechnique de Toulouse, France, 242 p.

22. VISSAC B., 2001. Les vaches de la République. Saisons et raisons d'un chercheur citoyen. Versailles, France, Inra Editions. (Coll. Espaces ruraux)

23. WOLTER R., 1992. Erreurs alimentaires et infertilité de la vache laitière. Prod. Lait. mod., 219 : 92-96.

24. WRIGHT I.A., RUSSEL A.J.F., HUBTER E.A. 1986. The use of body condition scoring to ration beef cows in late pregnancy. Anim. Prod., 43: 391-396.

Reçu le 26.11.2001, accepté le 07.08.2003

\section{Summary}

Madani T., Hubert B., Vissac B., Casabianca F. Analysis of Cattle Husbandry and Farming Systems Changes in an Algerian Sylvopastoral Area

This study analyzed the cattle husbandry role in livestock systems and farms in a forested area of Northeastern Algeria. The study area was a representative sample of Algeria 500,000 ha of cork oak (Quercus suber) forests. It is the wettest region of the country and it hosts the largest portion of Algeria suckling cattle herd. The authors first presented the framework of their study, information sources and animal husbandry types. Then, they developed a method to assess breeding and growth performances. The methodological approach to analyze livestock systems focused on interactions between female breeding profiles, animal genetic types and dynamics of animal body condition, which appeared as a reliable indicator that could be used as a diagnosis tool to control breeding. Comparison of animal husbandry practices with changes in animal body requirements and pastoral resources showed there were periods during the year (socalled saison-pratique) that were essential to analyze the adjustments implemented and their incidence on animal performances. Therefore, this study on the practices and evolution of livestock systems enabled the authors to identify new more relevant and operating categories of livestock systems that better reflected current trajectories. These categories helped produce reference frames that were adapted to the context and which could be used without the need to apply merely intensification paradigms.

Key words: Cattle - Reproductive performance - Body condition - Animal performance - Animal husbandry method - Agrosylvopastoral system - Algeria.

\section{Resumen}

Madani T., Hubert B., Vissac B., Casabianca F. Análisis de la actividad de la cría bovina y transformación de los sistemas de producción en situación silvo patoril en Argelia

El presente estudio analiza el papel jugado por la cría bovina en los sistemas de cría y las explotaciones de una cadena forestal en el nordeste argelino. La cadena es representativa de las 500000 ha de la sub área argelina que constituye la parte mas irrigada y alberga la mayor parte del hato bovino de leche del país. Después de presentar el cuadro de estudio, las fuentes de información y los tipos de establecimiento, los autores tratan los rendimientos de reproducción y de crecimiento de los bovinos. Este gesto metodológico del análisis de los sistemas de cría insistió sobre las inter acciones entre los perfiles de reproducción de las hembras, el tipo de material animal utilizado y la dinámica del estado corporal, el cual se revela un indicador fiable, pudiendo constituir un instrumento de diagnóstico para el control de la reproducción. La confrontación de las prácticas con la evolución de las necesidades de los animales y los recursos pastoriles permitió indicar los períodos, Ilamados estación-práctica, con el fin de analizar los ajustes establecidos y su incidencia sobre los rendimientos. De la misma manera, el estudio de las prácticas de la evolución de los sistemas de cría permitió elaborar categorías de sistemas de producción más pertinentes y operacionales, para evidenciar las trayectorias en curso. El presente estudio contribuyó así a la producción de los marcos de referencia adaptados a la situación, pudiendo ser utilizados sin tener que recurrir únicamente a los paradigmas de intensificación.

Palabras clave: Ganado bovino - Reproductividad - Condición corporal - Desempeño animal - Método de crianza Sistema agrosilvopascicolas - Argelia. 